\title{
Takotsubo Cardiomyopathy Associated with High-Grade Atrioventricular Block and Ventricular Fibrillation: A Case Report
}

Pedro Henrique de Santana

Fábio Américo Pedreira

Paulo Rogério Soares

Thiago Luis Scudeler (D)

Emergency Department, Instituto do Coração (InCor), Hospital das Clínicas da Faculdade de Medicina da Universidade de São Paulo, São Paulo, Brazil
Correspondence: Thiago Luis Scudeler Instituto do Coração (InCor), Hospital das Clínicas da Faculdade de Medicina da Universidade de São Paulo, Avenida Doutor Enéas de Carvalho Aguiar 44, AB, Sala II4, Cerqueira César, São Paulo, 05403-000, Brazil

Email thiago.scudeler@fm.usp.br

\begin{abstract}
Takotsubo cardiomyopathy (TCM) is a condition characterized by reversible left ventricular dysfunction. TCM usually has a good prognosis but, in rare situations, it can be associated with life-threatening arrhythmias. We report a case and the management of TCM with a high-grade AV block and QT prolongation followed by ventricular fibrillation.
\end{abstract}

Keywords: takotsubo cardiomyopathy, high-grade atrioventricular block, ventricular fibrillation, permanent pacemaker

\section{Introduction}

Takotsubo cardiomyopathy (TCM) is a form of nonischemic cardiomyopathy characterized by reversible changes in left ventricular function, especially in the midapical segments. ${ }^{1}$ The presence of arrhythmias in patients with TCM is uncommon, and high-grade AV block is very infrequent. ${ }^{2}$ However, the development of arrhythmias in a patient with TCM is associated with high morbidity and mortality. ${ }^{2}$ Although TCM usually reverses completely, AV block may persist and require a permanent pacemaker. ${ }^{3}$ We describe a case of TCM with high-grade AV block and QT prolongation followed by ventricular fibrillation (VF) cardiac arrest.

\section{Case Presentation}

A 56-year-old female with systemic arterial hypertension and grade 2 obesity, regularly using spironolactone $25 \mathrm{mg}$ once daily, losartan 50mg twice daily, and amlodipine $5 \mathrm{mg}$ twice daily, was admitted to the emergency department with dyspnea and adynamia for 1 day. The patient denied syncope, angina, or palpitations. She reported having experienced an episode of intense emotional stress 2 days before hospital admission. On examination, she had a heart rate of 50 beats per minute, blood pressure of 140/ $90 \mathrm{~mm} \mathrm{Hg}$, arterial oxygen saturation of $97 \%$ normal, and absence of clinical signs of heart failure. The electrocardiogram (ECG) on admission (Figure 1A) showed seconddegree AV block 2:1, right branch block, left anterior fascicular block, biphasic T wave in DII, DIII, aVF, V1, and V2 and inverted, deep, and asymmetric in DI, aVL, V3, V4, V5, and V6, with QRS duration of $142 \mathrm{~ms}$ and corrected QT interval of $689 \mathrm{~ms}$. Ultrasensitive troponin I was $396 \mathrm{ng} / \mathrm{L}$ (normal range, $\mathrm{NR}<40 \mathrm{ng} / \mathrm{L}$ ), and BNP was $307 \mathrm{pg} / \mathrm{nL}(\mathrm{NR}<100 \mathrm{pg} / \mathrm{mL})$. Renal parameters, serum electrolytes, and thyroid function tests were within normal limits. Transthoracic echocardiography (Figure 2 and Video) showed a left ventricular ejection fraction (LVEF) of $40 \%$, basal 

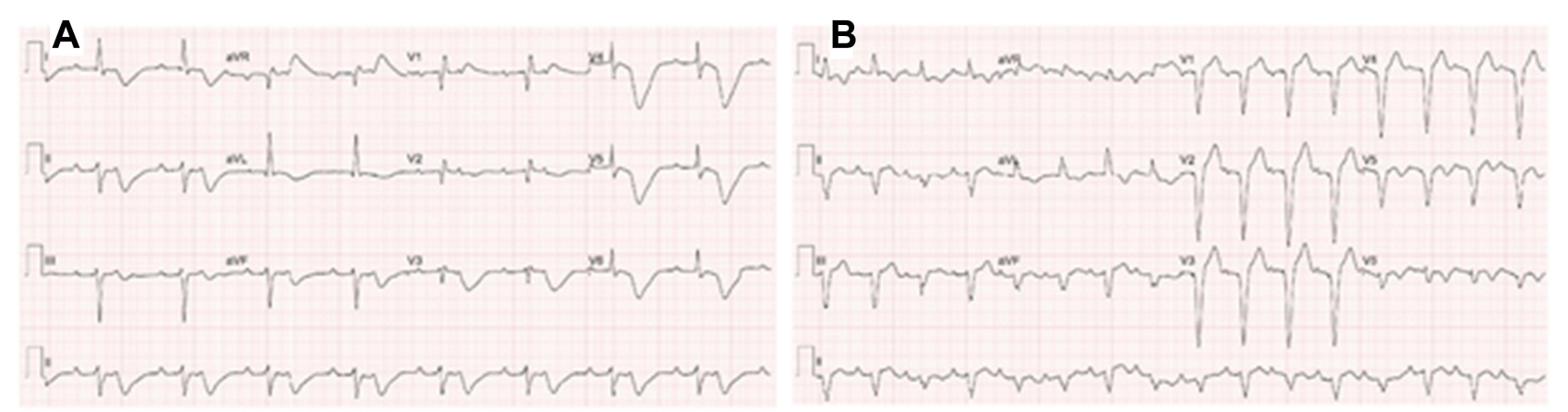

Figure I 12-Lead ECG (A) at admission showing incomplete right branch block, left anterior-superior divisional block, left axis deviation of the QRS, 2: I AV block, corrected QT interval prolongation $(689 \mathrm{~ms})$ and inverted T waves; (B) at discharge showing resolution of T wave inversions and shortening of the QT interval after pacemaker implantation.
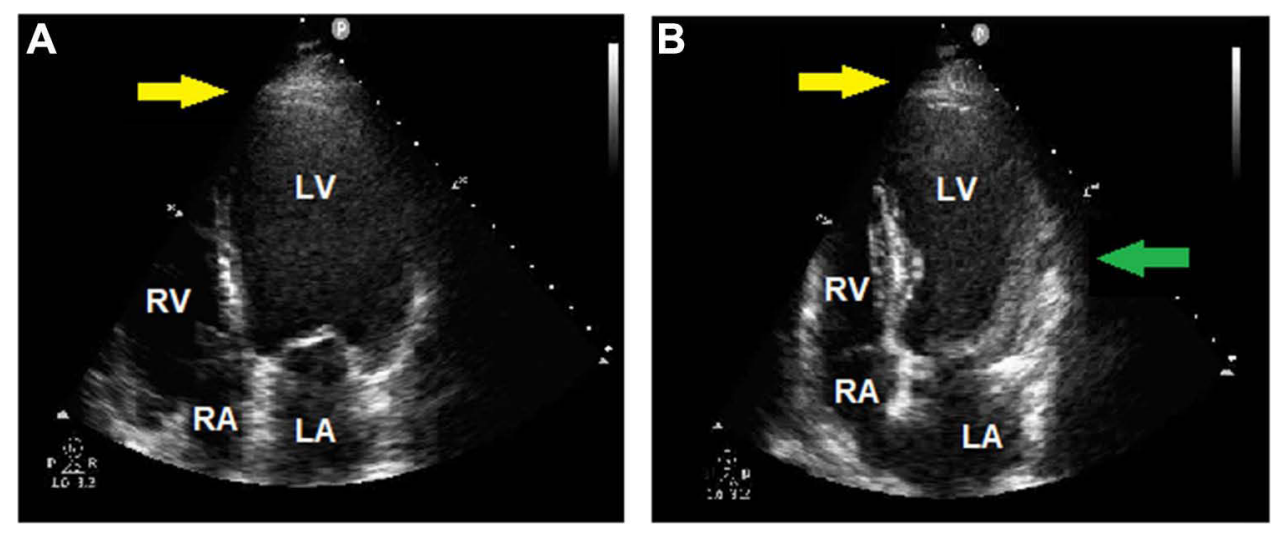

Figure 2 Transthoracic echocardiographic 4-chamber view of the left ventricle in diastole (A) and in systole (B) shows basal hypercontractility (green arrow) and midapical ballooning (yellow arrow).

Abbreviations: LA, left atrium; LV, left ventricle; RA, right atrium; RV, right ventricle.

hypercontractility and midventricular and apical ballooning, the most common form of TCM presentation. To expand on the investigation, coronary angiotomography did not show coronary lesions, with a calcium score of zero, and cardiac magnetic resonance (CMR) imaging confirmed the echocardiographic findings and did not show late enhancement (Figure 3). Initially, no transvenous pacemaker was indicated, because the patient was stable, asymptomatic, and had adequate ventricular escape $(50 \mathrm{bpm})$. On the same day, the patient experienced a VF cardiac arrest, and promptly underwent defibrillation with 200 joules and cardiopulmonary resuscitation maneuvers following the Advanced Cardiovascular Life Support protocol. She returned to spontaneous circulation after 3 minutes, with immediate recovery of consciousness. The post-cardiac arrest ECG showed the same pattern as that at the beginning of hospitalization. The patient did not receive tracheal intubation or sedo-analgesic drugs. Laboratory tests after cardiac arrest did not reveal electrolyte abnormalities. The patient underwent temporary pacemaker implantation, maintaining hemodynamic stability, without the need for vasoactive drugs. Because the high-grade AV block did not revert even after 14 days, the patient underwent a dual chamber permanent pacemaker implantation (DDD). On the 18th day, a transthoracic echocardiogram follow-up showed normalization of left ventricular wall motion, with an ejection fraction of $52 \%$; therefore, the patient was discharged from our hospital. ECG at hospital discharge showed complete resolution of T wave inversions and shortening of the QT interval (Figure 1B). At 1-year follow-up, ECG with pacing-off revealed the persistence of high-grade AV block but with a normal corrected QT interval.

\section{Discussion}

TCM was first described in 1990 by Sato et $\mathrm{al}^{4}$ and is characterized by transient left ventricular dysfunction, with clinical, electrocardiographic, and laboratory characteristics similar to those of acute coronary syndrome. The 

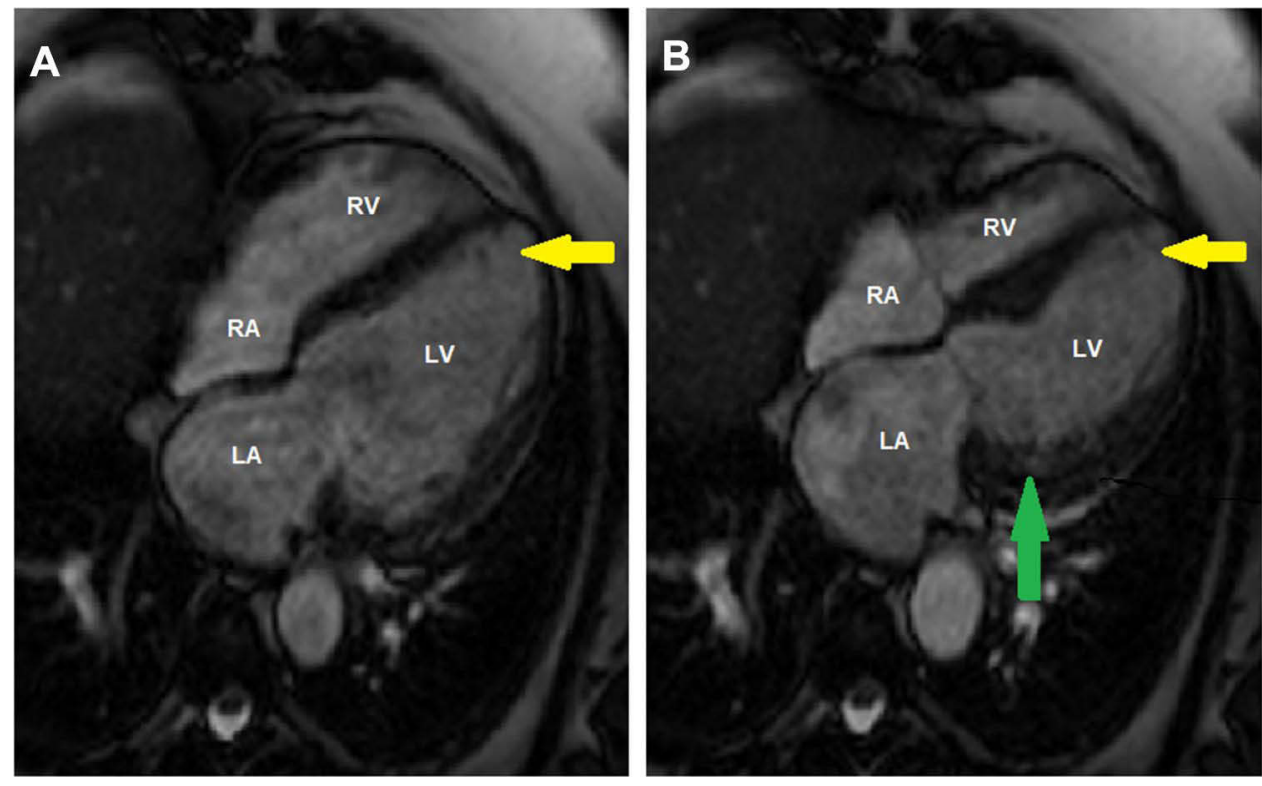

Figure 3 Cardiac magnetic resonance 4-chamber view of the left ventricle in diastole (A) and in systole (B) showing hypercontractility (green arrow) and typical apical ballooning (yellow arrow) in takotsubo syndrome.

Abbreviations: LA, left atrium; LV, left ventricle; RA, right atrium; RV, right ventricle.

syndrome occurs predominantly in postmenopausal women $^{1}$ after an episode of physical or psychological stress. Its pathophysiology is not yet fully understood, but excess catecholamines have been postulated to be central to the pathogenesis of TCM. ${ }^{5}$

The diagnosis is based on clinical, echocardiographic, and laboratory data. Although this is not a typical description of the syndrome, the patient's clinical condition follows the diagnostic criteria proposed by the Mayo Clinic in 2008, which include (1) transient hypokinesis, akinesis, or dyskinesis of the left ventricular mid segments with or without apical involvement; the regional wall-motion abnormalities extend beyond a single epicardial vascular distribution; (2) absence of obstructive coronary disease or angiographic evidence of acute plaque rupture; (3) new electrocardiographic abnormalities (either ST-segment elevation and/or T-wave inversion) or modest elevation in cardiac troponin; and (4) absence of all of the following: pheochromocytoma, myocarditis, and hypertrophic cardiomyopathy. ${ }^{6}$

The clinical suspicion was made based on the changes in segmental mobility detected on the transthoracic echocardiogram. This investigation was further developed with the CMR, which confirmed the changes in segmental mobility and the suspicion of TCM. In this sense, CMR also lends itself to the investigation of differential diagnoses, such as myocarditis or even acute myocardial infarction. According to the description by Eitel et al, ${ }^{7}$ the observation of late gadolinium enhancement allows such differentiation, because it is absent in TCM, intense in acute myocardial infarction (greater than 5 standard deviations above the mean of the intensity of the myocardial signal), and irregular in myocarditis. In some cases of TCM, it is possible to observe myocardial edema in CMR; however, this finding is considered nonspecific.

We opted for coronary tomography instead of coronary angiography to rule out coronary artery disease (CAD), because the pretest probability of CAD for our patient was low and the clinical and echocardiographic diagnosis of TCM was very likely.

ECG is altered in most patients with TCM (>95\%) and usually shows ST segment elevation in the precordial leads (81\%), ST segment depression, T-wave inversion (usually deep and diffuse), prolongation of the QT interval, and presence of pathological Q waves. ${ }^{8}$ Arrhythmias have been described as an uncommon complication of TCM and may include ventricular arrhythmia, atrial fibrillation, torsades de pointes, and AV node dysfunction. ${ }^{9}$ High-grade AV block is rarely found in patients with TCM and has a prevalence of only $2.9 \%{ }^{9}$ The exact association between AV block and TCM is not clear, but it can be explained by the continual ischemic status due to microvascular dysfunction. ${ }^{10}$

Our case shows a rare association between AV block and TCM. Although recovery of left ventricular function occurs in most patients, AV block can persist. ${ }^{11}$ It is 
difficult to know whether AV block is the cause of or the result of stress-induced cardiomyopathy. Therefore, the best method for managing high-grade AV block associated with TCM remains uncertain. The electrical changes present in this patient (mainly the prolongation of the QT interval) may have favored the outcome for VF (phenomenon $\mathrm{R}$ over $\mathrm{T}$ ). The dramatic evolution to VF suggests that the implantation of a temporary pacemaker in these patients should not be delayed. An implantable cardioverter defibrillator was not indicated because VF can be managed with a temporary approach until recovery of repolarization time and left ventricular function. ${ }^{10}$ In our patient, we opted for the implantation of a permanent pacemaker on day 14 due to the persistence of AV block. In patients with TCM, time interval pacemaker implantation is not well established. ${ }^{12}$ In some cases, AV conduction disorder is transient. However, temporary pacemaker implantation for a long time can be associated with several complications, such as perforation of the right ventricle and infections. Long-term follow-up is necessary to verify the resumption of $1: 1 \mathrm{AV}$ conduction and, therefore, unnecessary stimulation should be avoided.

In Box 1 you will find the learning points of this case report.

\section{Box I Learning Points}

I. The presence of arrhythmias in patients with TCM is uncommon, and high-grade AV block is associated with high morbidity and mortality.

2. The exact association between AV block and TCM is not clear, but it can be explained by the continual ischemic status due to the microvascular dysfunction.

3. TCM usually reverses completely, but AV block may persist in spite of complete clinical recovery.

4. Patients with high-grade AV block and TCM, especially those with prolonged QT interval, require a temporary pacemaker due to the high risk of malignant ventricular arrhythmias.

5. The time required for the indication of the definitive pacemaker is not yet clear. Because the likelihood of AV block reversal is low, we believe that the time between symptom onset up to 14 days is adequate.

6. The choice of pacemaker (dual chamber versus biventricular) in patients with left ventricular dysfunction (LV) due to TCM is not clear. Because LV dysfunction is reversible in most patients with TCM, a dual chamber pacemaker may be preferable to a biventricular pacemaker.
In conclusion, the recognition of $\mathrm{AV}$ conduction defects in patients with TCM is important, because they may not resolve and may need an intervention like pacemaker implantation. Such situations are underrecognized but are potentially fatal and need timely management for patient safety.

\section{Abbreviations}

TCM, takotsubo cardiomyopathy; ECG, electrocardiogram; NR, normal range; LVEF, left ventricular ejection fraction; CMR, cardiac magnetic resonance; LV, left ventricular; $\mathrm{AV}$, atrioventricular; $\mathrm{VF}$, ventricular fibrillation.

\section{Data Sharing Statement}

All data generated or analyzed during this study are included in this published article.

\section{Ethics Approval and Consent to Participate}

The research protocol was approved by the Ethics Committee of the Instituto do Coração, Universidade de São Paulo. Written informed consent was obtained from the patient for publication of this case report.

\section{Author Contributions}

All authors made substantial contributions to the conception and design, acquisition of data, or analysis and interpretation of data; took part in drafting the article or revising it critically for important intellectual content; agreed to submit to the current journal; gave final approval of the version to be published; and agree to be accountable for all aspects of the work.

\section{Funding}

This research did not receive any specific grant from funding agencies in the public, commercial, or not-forprofit sectors.

\section{Disclosure}

The authors declare that they have no conflicts of interest.

\section{References}

1. Pilgrim TM, Wyss TR. Takotsubo cardiomyopathy or transient left ventricular apical ballooning syndrome: a systematic review. Int J Cardiol. 2008;124(3):283-292.

2. Patil SP, Gonuguntla K, Rojulpote C, Kumar M, Chen KG. Takotsubo cardiomyopathy related complete heart block: a nationwide cohort analysis. Eur Heart J. 2020;41(2):ehaa946-0697. 
3. Benouda L, Roule V, Foucault A, Dahdouh Z, Lebon A, Milliez P. Conduction disturbances in takotsubo cardiomyopathy: a cause or a consequence? Int J Cardiol. 2012;159(1):61-62.

4. Sato H, Tateishi H, Uchida T, et al. Takotsubo type cardiomyopathy due to multivessel spasm. In: Kodama K, Haze K, Hon M, editors. Clinical Aspect of Myocardial Injury: From Ischemia to Heart Failure. Tokyo: Kagaku Hyoronsha; 1990:56-64.

5. Wittstein IS, Thiemann DR, Lima JAC, et al. Neurohumoral features of myocardial stunning due to sudden emotional stress. $N$ Engl J Med. 2005;352(6):539-548.

6. Scantlebury DC, Prasad A. Diagnosis of Takotsubo cardiomyopathy. Circ J. 2014;78(9):2129-2139.

7. Eitel I, von Knobelsdorff-brenkenhoff F, Bernhardt P, et al. Clinical characteristics and cardiovascular magnetic resonance findings in stress (takotsubo) cardiomyopathy. JAMA. 2011;306(3):277-286.

8. Gianni M, Dentali F, Grandi AM, Sumner G, Hiralal R, Lonn E. Apical ballooning syndrome or Takotsubo cardiomyopathy: a systematic review. Eur Heart J. 2006;27(13):1523-1529.
9. Syed FF, Asirvatham SJ, Francis J. Arrhythmia occurrence with Takotsubo cardiomyopathy: a literature review. Europace. 2011;13 (6):780-788

10. Inayat F, Virk HUH, Ullah W, Riaz I. Takotsubo cardiomyopathy-related complete heart block and torsades de pointes. BMJ Case Rep. 2017;2017:bcr2016218017.

11. Kodama S, Miyoshi K, Shiga Y, et al. Takotsubo cardiomyopathy complicated by high-grade atrioventricular block: a report of two cases. Exp Clin Cardiol. 2009;14:e35.

12. Le MT, Grimard C, Varenne O, Jakamy R, Rosencher J. Tako-Tsubo cardiomyopathy and high-degree atrio-ventricular block: do we need to wait left ventricular function recovery to implant a pacemaker? Int J Cardiol. 2014;176(2):e56-e59.

\section{Publish your work in this journal}

The International Medical Case Reports Journal is an international, peer-reviewed open-access journal publishing original case reports from all medical specialties. Previously unpublished medical posters are also accepted relating to any area of clinical or preclinical science. Submissions should not normally exceed 2,000 words or 4 published pages including figures, diagrams and references. The manuscript management system is completely online and includes a very quick and fair peer-review system, which is all easy to use. Visit http://www.dovepress.com/testimonials.php to read real quotes from published authors. 